\title{
Spinal Cord Compression Secondary to Metastatic Sinonasal Undifferentiated Carcinoma
}

\author{
Arthur Wang ${ }^{a} \quad J o h n$ V. Wainwright ${ }^{a} \quad$ Anubhav G. Amin ${ }^{a} \quad$ Eric Vail $^{b}$ \\ Rachel Silverstein ${ }^{c}$ Kaushik Das ${ }^{a}$ \\ ${ }^{a}$ Department of Neurological Surgery, New York Medical College, Westchester, NY, USA; \\ ${ }^{b}$ Department of Pathology, New York Medical College, Westchester, NY, USA; \\ 'Department of Orthopedic Surgery, New York Medical College, Westchester, NY, USA
}

\section{Keywords}

Spinal cord compression · Metastasis · Sinonasal undifferentiated carcinoma

\begin{abstract}
Sinonasal undifferentiated carcinoma (SNUC) is a rare malignancy of the upper airways and anterior skull base that carries a poor prognosis. The tumor is known to be invasive into the surrounding structures of the skull base and brain. To date, there is only one existing case report documenting drop metastasis to the intradural extramedullary spinal cord. To the best of our knowledge, we present the second case of metastatic SNUC to the spine. This report describes a 59-year-old male with a history of head and neck SNUC who presented with thoracic back pain and bilateral lower extremity paresis. Neuroimaging demonstrated an extradural thoracic mass with severe spinal cord compression. The patient underwent thoracic laminectomy and fusion for decompression of the spinal cord and internal stabilization. The pathology returned as SNUC. The patient was subsequently lost to follow-up from our institution. Metastatic SNUC is rare. We discuss the relevant clinical imaging and review the literature. Such a malignancy portends a very poor prognosis.

(C) 2017 The Author(s)

Published by S. Karger AG, Basel
\end{abstract}




\section{Case Reports in Oncology}

Wang et al.: Spinal Cord Compression Secondary to Metastatic Sinonasal Undifferentiated Carcinoma

\section{Introduction}

Sinonasal undifferentiated carcinoma (SNUC) is a locally aggressive tumor of the paranasal sinuses and nasal cavity [1]. Frierson et al. [2] first described this tumor in 1986 and since then, only 150 cases of this entity have been reported in the literature. The tumor is locally aggressive, invading the skull base and intracranial structures. It can show distant metastasis, the most common sites being the cervical lymph nodes, lung, bone, and liver $[3,4]$.

We report a case of metastatic SNUC to the extradural spinal cord causing severe cord compression in a patient with a known diagnosis of SNUC. To date, there exists only one other case report of metastatic SNUC to the intradural extramedullary spine [1]. We describe the clinical and imaging features and review the existing literature on this rare pathology.

\section{Case Report}

A 59-year-old male presented with 5 days of difficulty with ambulation secondary to progressively worsening back pain and bilateral lower extremity weakness and numbness. The patient denied upper extremity weakness, saddle anesthesia, or bowel and bladder dysfunction. The patient's past medical history was significant for coronary artery disease, hypertension, and cholecystectomy, and he was status post excisional biopsy of an infiltrating SNUC located in the clivus and extending into the left petrous apex 2.5 years prior to the current presentation (Fig. 1). The patient was status post chemotherapy with cisplatin and etoposide and radiation therapy, but had subsequently been lost to follow-up.

Physical examination was significant for $0 / 5$ motor strength in bilateral lower extremities, with associated decreased sensation to light touch and pinprick. Magnetic resonance imaging of the thoracic spine revealed a $6.4 \times 3.2 \mathrm{~cm}$ mixed-intensity right-sided extradural extramedullary lesion extending from T7 to T9 with complete compression of the spinal cord at these levels (Fig. 2, Fig. 3).

The patient underwent T8 vertebrectomy, decompressive laminectomy, resection of the epidural tumor, placement of a vertebral body cage, and posterior segmental instrumentation from T6 to T11 (Fig. 4). Histopathologic examination revealed the tumor to be a carcinoma. Microscopic examination showed nodules of small basaloid cells arranged in anastomosing cords (reticular pattern) in a rich myxoid/mucoid stroma (Fig. 5). The neoplastic cells showed malignant cytological features, including irregular, moderately pleomorphic nuclei, significantly sized nucleoli, limited cytoplasm, a brisk amount of mitotic activity, and focal apoptosis. One small area of focal squamous differentiation was seen, otherwise no formation of true luminal/glandular structures and no lobular configuration with peripheral palisading of tumor cells or keratinization was observed. The stroma was positive for alcian blue and mucicarmine. The immunohistochemical study showed that the neoplastic cells were diffusely and strongly positive for AE1/AE3, cytokeratin 5/6, vimentin, p63, and p40 (Fig. 6). All of the neoplastic cells displayed strong nuclear positive reaction for EBV (EBERISH). Negative immunomarkers included S100, calponin, CD117, and epithelial membrane antigen. 
 Oncology}

Wang et al.: Spinal Cord Compression Secondary to Metastatic Sinonasal Undifferentiated Carcinoma

\section{Discussion}

There are three histological subtypes of sinonasal malignancies with neuroendocrine features: olfactory neuroblastoma, SNUC, and sinonasal neuroendocrine carcinoma [5]. These malignancies can only be diagnosed through histological analysis.

The World Health Organization defines SNUC as an aggressive tumor of the paranasal sinuses and nasal cavity composed of pleomorphic undifferentiated tumor cells with necrosis [6]. SNUC comprises $<1 \%$ of all malignancies. The tumor typically grows rapidly and is locally invasive, with extension to the orbits, anterior skull base, and intracranial structures. The exact pathogenesis is still unclear; however, it is believed that the carcinoma arises from malignant epithelial cells in the nasal mucosa [2]. Since the tumor arises in the nasal cavity, patients commonly present with nonspecific symptoms such as nasal obstruction and epistaxis, often leading to a delay in diagnosis [7]. A few occupational risk factors for SNUC have been identified, including furniture workers exposed to wood dust and nickel refinery workers exposed to nickel. The association with smoking is unclear.

The imaging of primary SNUC is similar to that of neuroendocrine carcinoma, lymphoepithelioma, olfactory neuroblastoma, lymphoma, and rhabdomyosarcoma. As a result, diagnosis is through an excisional biopsy. As in our patient, histology typically shows nests of undifferentiated cells without structural differentiation. Evidence of high mitotic activity with areas of necrosis and dark prominent nucleoli and vascular invasion is commonly seen. Immunohistochemistry typically shows positivity for the epithelial markers cytokeratin and epithelial membrane antigen [8].

Metastatic spinal cord compression is an oncological emergency that requires prompt treatment. Our patient and the previously described case in the literature highlight a few important points.

First, what is the optimal timing of surgery in a case of metastatic SNUC with spinal cord compression? Timing depends on the clinical condition of the patient. Patients with complete spinal cord injury have a very poor prognosis for neurological recovery even after surgical decompression and stabilization. Meanwhile, incomplete spinal cord injury patients have significantly better neurological outcome if surgical decompression is performed within $48 \mathrm{~h}$ of acute presentation of symptoms $[9,10]$.

Second, what is the pathogenesis of spinal cord metastasis? Tumors can spread to the spine through four ways [11-13]. Hematogenous dissemination via the anterior spinal, segmental, and radiculomedullary arteries can lead to metastatic disease of the vertebral body or spinal cord substance. Next is venous dissemination via Batson's paravertebral plexus, which connects the venous anatomy of the spinal cord with the veins of the head, neck, and thoracoabdominal wall. The veins that make up Batson's plexus are valveless and located in the epidural space. Tumor spread through these veins typically results in epidural metastatic disease. Tumors can also directly invade via local extension to the spine, vertebral column, or nerve roots. Last is spread through the cerebrospinal fluid, which leads to intradural metastasis. In our patient, the metastatic disease was epidural and so we did not pursue any spinal fluid sampling.

Third, what is the optimal treatment for metastatic SNUC? Current treatment favors a multimodality approach with surgical resection, chemotherapy, and/or radiation therapy. One case series reported a 3-year survival rate of $67 \%$ in patients undergoing gross total surgical resection with adjuvant radiation or chemotherapy [14]. Similarly, another case series reported a 5-year survival rate of $74 \%$ when surgical resection was combined with radiation or chemotherapy for resectable lesions [15]. 
 Oncology}

Wang et al:: Spinal Cord Compression Secondary to Metastatic Sinonasal Undifferentiated Carcinoma

\section{Conclusions}

This case report adds to the existing literature of squamous cell carcinoma secondary to metastatic SNUC. There are only a handful of case reports documenting metastasis, with only one previous case report describing metastasis to the intradural spine. Because of the rarity of such tumors, the pathogenesis and optimal treatment modalities remain elusive. Despite more common etiologies of squamous cell carcinoma, this case report highlights the importance of being mindful of rare diseases.

\section{Statement of Ethics}

The authors have no ethical conflicts to disclose.

\section{Disclosure Statement}

The authors declare that there are no conflicts of interest.

\section{References}

1 Ghosh S, Weiss M, Streeter O, Sinha U, Commins D, Chen TC: Drop metastasis from sinonasal undifferentiated carcinoma. Spine 2001;26:1486-1491.

-2 Frierson HF Jr, Mills SE, Fechner RE, Taxy JB, Levine PA: Sinonasal undifferentiated carcinoma. An aggressive neoplasm derived from schneiderian epithelium and distinct from olfactory neuroblastoma. Am J Surg Pathol 1986;10:771-779.

-3 Miyaguchi M, Sakai S, Takashima H, Hosokawa H: Lymph node and distant metastases in patients with sinonasal carcinoma. J Laryngol Otol 1995;109:304-307.

-4 Righi PD, Francis F, Aron BS, Weitzner S, Wilson KM, Gluckman J: Sinonasal undifferentiated carcinoma: a 10-year experience. Am J Otolaryngol 1996;17:167-171.

-5 Soldatova L, Campbell RG, Carrau RL, Prevedello DM, Wakely P Jr, Otto BA, Filho LF: Sinonasal carcinomas with neuroendocrine features: histopathological differentiation and treatment outcomes. J Neurol Surg B Skull Base 2016;77:456-465.

6 Frierson HF Jr: Sinonasal undifferentiated carcinoma; in Barnes L, Eveson JW, Reichart P, Sidransky D (eds): World Health Organization Classification of Tumours. Pathology and Genetics of Head and Neck Tumours. Lyon, IARC, 2005, p 19.

7 Khan MN, Konuthula N, Parasher A, Genden EM, Miles BA, Govindaraj S, Iloreta AM: Treatment modalities in sinonasal undifferentiated carcinoma: an analysis from the National Cancer Database. Int Forum Allergy Rhinol 2017;7:205-210.

8 Singh L, Ranja R, Arava S, Singh MK: Role of p40 and cytokeratin 5/6 in the differential diagnosis of sinonasal undifferentiated carcinoma. Ann Diagn Pathol 2014;18:261-265.

-9 Chaichana KL, Woodworth GF, Sciubba DM, McGirt MJ, Witham TJ, Bydon A, Wolinsky JP, Gokaslan Z: Predictors of ambulatory function after decompressive surgery for metastatic epidural spinal cord compression. Neurosurgery 2008;62:683-692.

10 Fürstenberg CH, Wiedenhöfer B, Gerner HJ, Putz C: The effect of early surgical treatment on recovery in patients with metastatic compression of the spinal cord. J Bone Joint Surg Br 2009;91:240-244.

$\checkmark 11$ Barolat-Romana G, Benzel EC: Spinal intradural extraarachnoid metastasis. Surg Neurol 1983;19:137143.

12 Batson OV: The function of the vertebral veins and their role in the spread of metastases. 1940. Clin Orthop Relat Res 1995;312:4-9.

13 Edelson RN, Deck MD, Posner JB: Intramedullary spinal cord metastases: clinical and radiographic findings in nine cases. Neurology 1972;22:1222-1231.

14 Tanzler ED, Morris CG, Orlando CA, Werning JW, Mendenhall WM: Management of sinonasal undifferentiated carcinoma. Head Neck 2008;30:595-599. 


\section{Case Reports in Oncology}

\begin{tabular}{l|l}
\hline Case Rep Oncol 2017;10:596-604 \\
\hline DOI: 10.1159/000477845 & $\begin{array}{l}\text { C } 2017 \text { The Author(s). Published by S. Karger AG, Basel } \\
\text { www.karger.com/cro }\end{array}$ \\
\hline
\end{tabular}

Wang et al:: Spinal Cord Compression Secondary to Metastatic Sinonasal Undifferentiated Carcinoma

15 Al-Mamgani A, van Rooij P, Mehilal R, Tans L, Levendag PC: Combined-modality treatment improved outcome in sinonasal undifferentiated carcinoma: single-institutional experience of 21 patients and review of the literature. Eur Arch Otorhinolaryngol 2013;270:293-299.

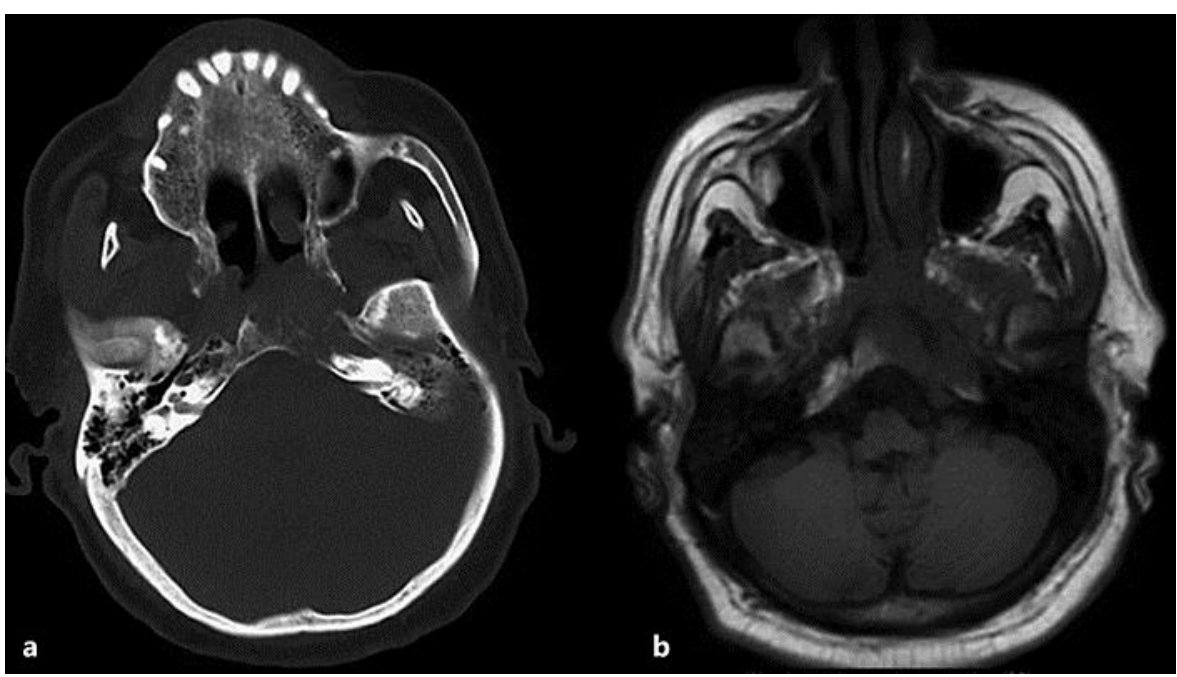

Fig. 1. Axial computed tomography (a) and T2-weighted magnetic resonance imaging (b) of the head demonstrating a destructive lytic left-sided clival based mass with extension into the left petrous apex. 


\section{Case Reports in Oncology}
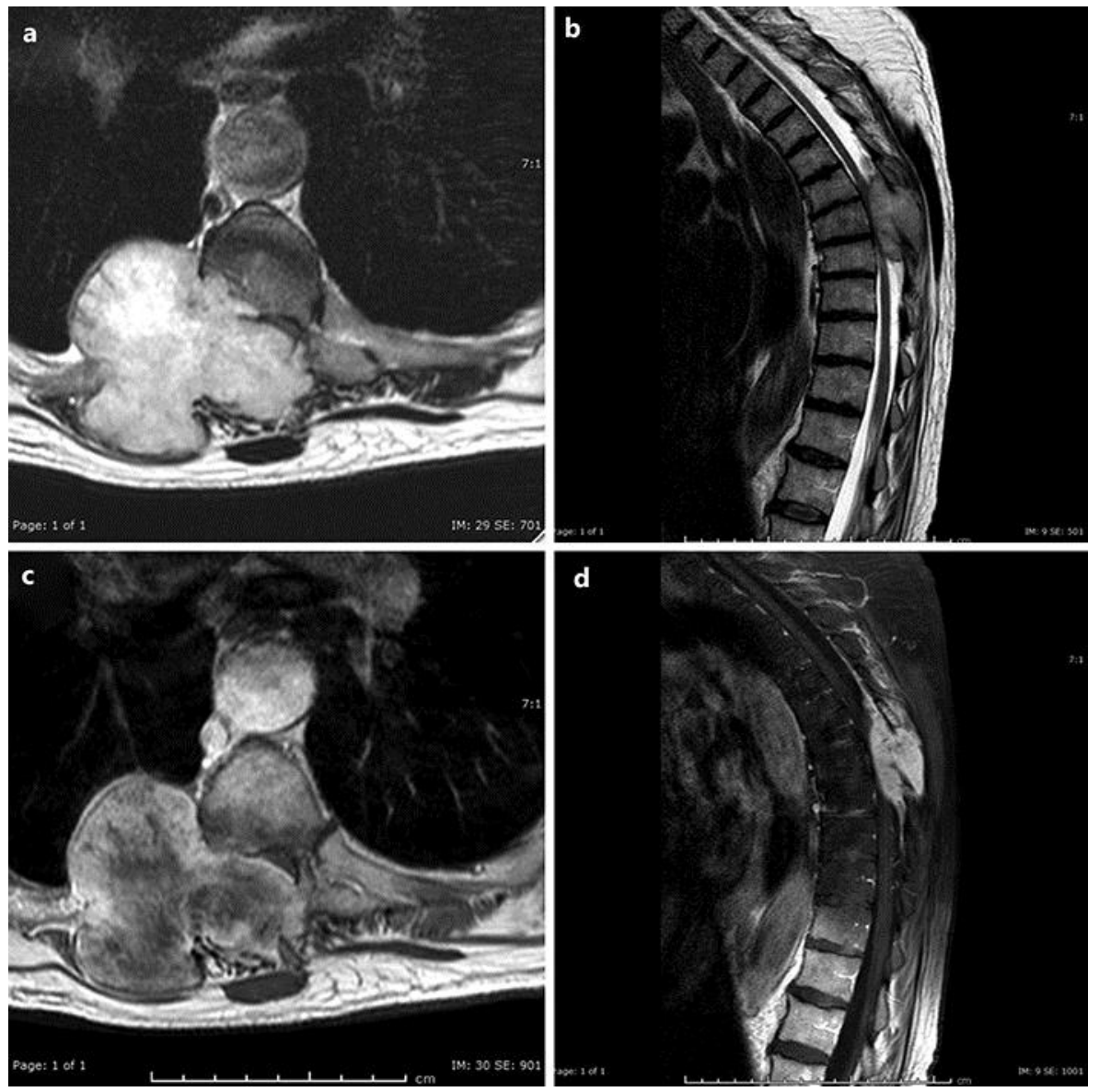

Fig. 2. a, b Representative axial (a) and sagittal (b) T2-weighted magnetic resonance imaging of the thoracic spine demonstrating the large, lobular, right paraspinal mass centered at the costovertebral junction causing significant bony erosion and severe, high-grade epidural spinal cord compression with extension into the pleural space. c, d Gadolinium-enhanced axial (c) T1-weighted and sagittal (d) T1-weighted and fat-suppressed magnetic resonance imaging of the thoracic spine again demonstrating the large paraspinal mass with avid contrast enhancement and invasion of the pleural space. 


\section{Case Reports in Oncology}

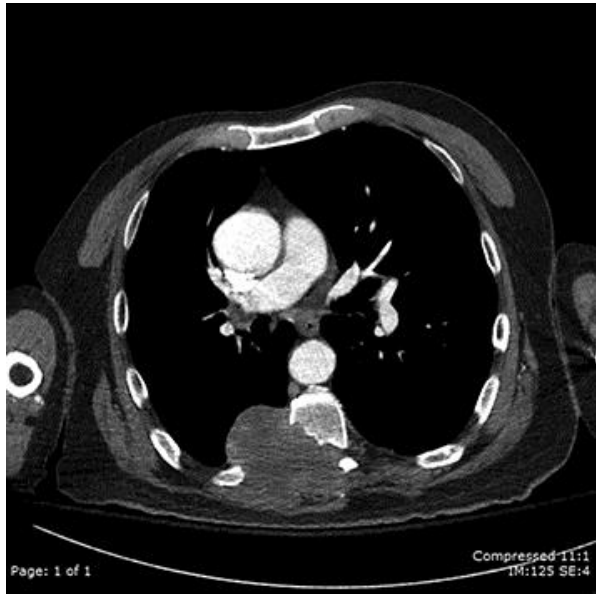

Fig. 3. Axial contrast-enhanced chest computed tomography at the T8 level demonstrating a large mass in the right paraspinal region with significant bony erosion of the adjacent ribs and vertebral body, invasion of the spinal canal, and extension into the pleural space.
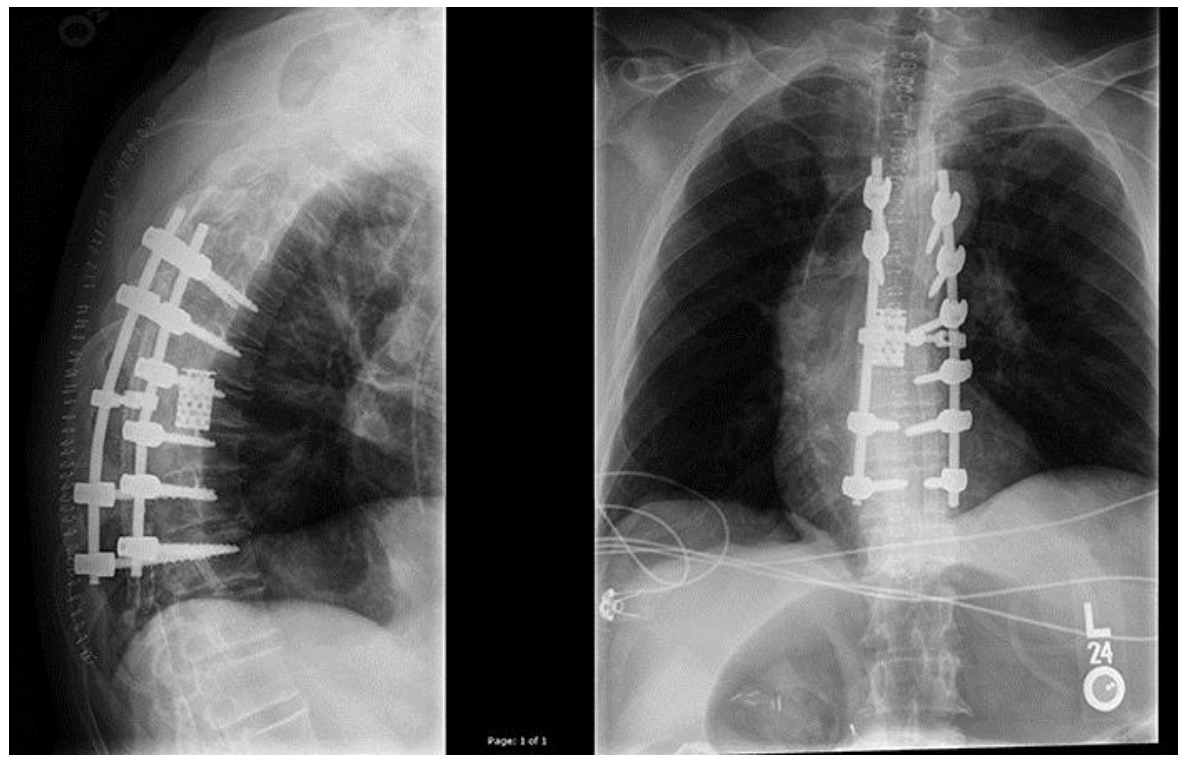

Fig. 4. Anteroposterior and lateral X-rays of the thoracic spine demonstrating the hardware construct following the posterior, lateral extracavitary approach for tumor resection and partial T8 corpectomy with T6-T11 instrumented fusion. 


\section{Case Reports in Oncology}

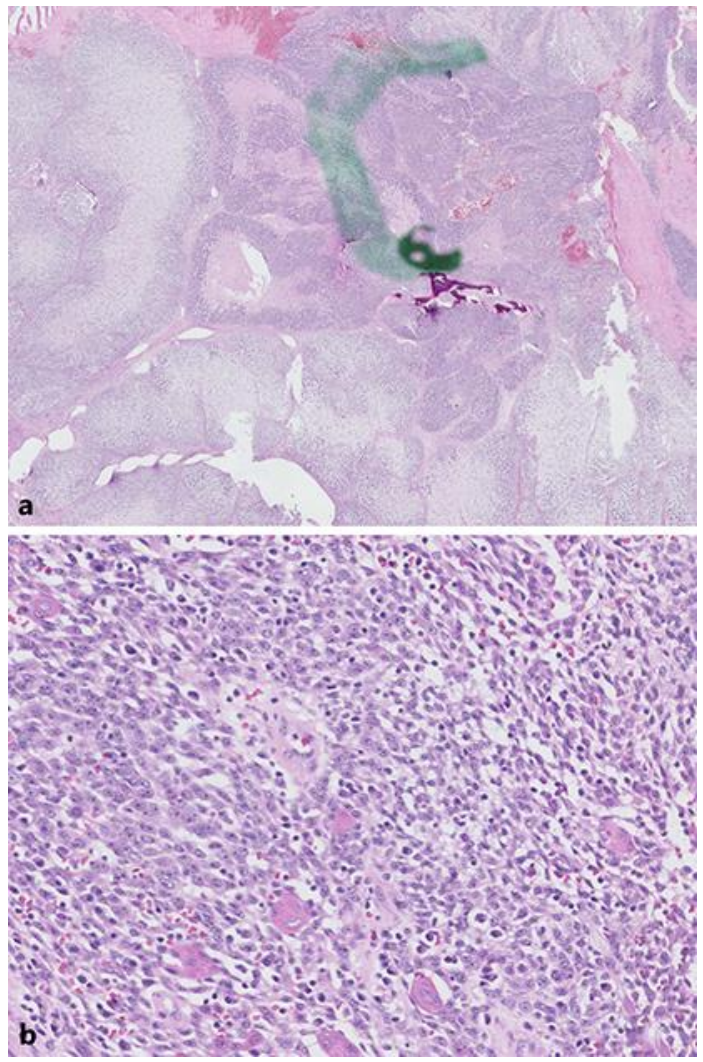

Fig. 5. Light photomicrographs of the surgical specimen with hematoxylin and eosin staining. a Low-power magnification demonstrates nests and chords of epithelioid cells interspersed with areas of spindle cells in a chondromyxoid matrix. b High-power magnification demonstrates the tumor cells with high nuclear to cytoplasmic ratio, moderate mitotic activity, and prominent nucleoli. 


\section{Case Reports in Oncology}

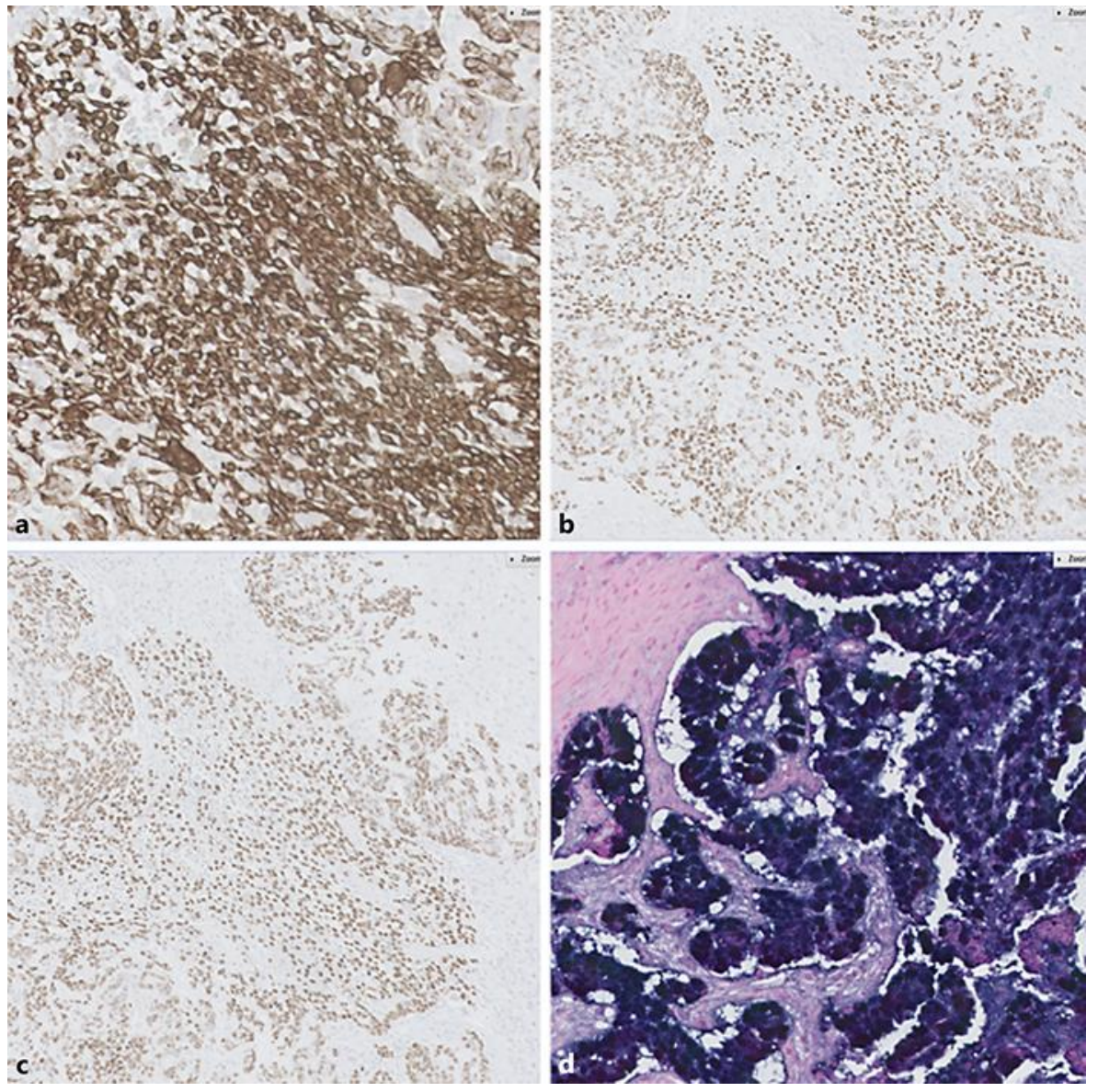

Fig. 6. High-power photomicrograph showing positive immunohistochemical staining for cytokeratin 5/6 (a), p40 (b), p63 (c), and EBV (d). 